\title{
Téoros
}

Revue de recherche en tourisme

\section{La mer, le littoral et la culture se conjuguent}

L'étude de cas de Bouctouche, ville acadienne

\section{Marc LeBlanc}

Volume 20, numéro 1, printemps 2001

Mers et littoraux

URI : https://id.erudit.org/iderudit/1071911ar

DOI : https://doi.org/10.7202/1071911ar

Aller au sommaire du numéro

Éditeur(s)

Université du Québec à Montréal

ISSN

0712-8657 (imprimé)

1923-2705 (numérique)

Découvrir la revue

Citer cet article

LeBlanc, M. (2001). La mer, le littoral et la culture se conjuguent : l'étude de cas de Bouctouche, ville acadienne. Téoros, 20(1), 49-55.

https://doi.org/10.7202/1071911ar d'utilisation que vous pouvez consulter en ligne.

https://apropos.erudit.org/fr/usagers/politique-dutilisation/ 


\section{La mer, le littoral et la culture se conjuguent L'étude de cas de Bouctouche, ville acadienne}

\section{Marc LeBlane}

\begin{abstract}
Le Nouveau-Brunswick a déployé de nombreux efforts de commercialisation et de développement de produits touristiques dans le but d'ativer les touristes desireux de faire un séjour à la plage. La province propose, surtout le long du litoral acadien, plus de $200 \mathrm{~km}$ de plages accessibles aux visiteurs, sant de sites sans aucun service à des endroits où l'on peut pratiquer la baignade en toute sécurité, en plus d'avoir accès à des passerelles, à des services sanitaires et à des stationnements adéquats. Encore faut-il que soient développés de nouveaux produits, tels l'écotourisme, les activités éducatives et le maillage entre la nature et la culture, pour des clientèles en pleine émergence.
\end{abstract} dont certaines ont les \& eaux salées. les plus chaudes au nord de la Virginie s (Gow du N, B., 2000). Les plages qui attivent le plus grand nombre de touristes se siment au sud-est de la province dans la region du Parc national Kouchibouguac, de Shédiac et de Cap-Pelé. Ces endroits offrent essentiellement le bain de soleil et la baignade. Tout en étant conscientes de la forte concurrence el du caractène saisomier de ce produit, l'ensemble des régions acadiennes du littoral s'inscrit dans ce positionnement et en tire d'importantes retombees économiques.
$\mathbf{N}$ ul doute que le produit plage de la province s'est grandement redressé au cours des deux dernières décennies, pas-

L'apparition et surtout la croissance rapide du tourisme de masse après la Seconde Guerre mondiale a provoqué la dégradation de l'environnement (Potts et Harrill, 1998 : 134). Au début des années 1970 , quelques auteurs (McHarg, 1969 ; Gunn, 1972) ont commencé à s'intéresser de près à un développement touristique harmonieux et * durable ${ }^{\|}$* en proposant des techniques d'aménagement respectueuses de l'environnement. Potts et Harril soulignent que malgré l'existence de ces nouvelles techniques, peu de place était réservée à la participation du public au processus de développement touristique (1998: 134), Ils ajoutent que la population locale étant peu informée des projets, cela laissait place à toutes sortes de spéculations parmi les habitants, ce qui menait à une perception très négative du tourisme. Cette attitude de la communauté d'accueil vis-à-vis le tourisme aboutissait à une insatisfaction du touriste qui finissait par bouder la région. La participation du public au développement a pris son véritable envol durant les annés 1980 et 1990 (Potts et Harril, 1998 : 135). D'ailleurs, Seekings insiste sur le fait que le développement durable touristique était devenu trop important pour laisser la décision finale uniquement aux experts et que la communauté devait aussi avoir son mot à dire $(1980: 253)$.

C'est au titre de la participation populaire, entre autres, que le développement de la région de Bouctouche suscite la curiosité des intervenants touristiques, des chercheurs et des environnementalistes d'un peu partout au Canada. Lack (1999) en faisait la remarque à peine deux années après l'ouverture de l'une des deux attractions principales de Bouctouche, petite ville acadienne de 2500 habitants située à $65 \mathrm{~km}$ au nord de Moncton et à $25 \mathrm{~km}$ de Shédiac.

Par l'entremise de l'étude de cas de Bouctouche, il est possible d'amorcer une réflexion sur l' hypothèse de Potts et Harril que l'on doit considérer le développement touristique durable comme un outil de développement social plutôt que la simplification de la nature par' l'homme. Ils ajoutent que c'est lá communauté qui doit être \& durable * et non le tourisme (1998: 140). Par conséquent, le présent article a pour but de présenter le développement du littoral et d'en exposer les diverses retombées sur l'ensemble de la communauté. Dans un premier temps, il s'agira de décrire les principaux êléments qui ont incité cette communauté à se prendre en main à partir d'une expérience de développement touristique axé sur la culture. Par la suite, les grandes étapes du développement du projet écotouristique de la baie de Bouctouche seront abordées, Enfin, les retombées majeures sur cette région seront présentées ainsi que des commentaires sur ce que réserve l'avenir. 


\section{Une tentative de développement culturel se terminant par un plan de tourisme durable}

La ville de Bouctouche est connuc à divers titres, à la fois positifs et négatifs. Elle a vu naître des personnages connus dans tout le Canada et au-delà des frontières, tels K.C. Irving, fondateur de l'entreprise familiale multimilliardaire, et Antonine Maillet, devenue célèbre dans la francophonie mondiale après avoir reçu le prix Goncourt en 1979. Toutefois dans les années 1970 , la région de Kent, où se situe Bouctouche, était considérée comme la deuxième région la plus pauvre au Canada (Savoie, 1992). Les intervenants écono= miques de Bouctouche, tout comme ceux de toute la région d'ailleurs, ont alors convenu que pour se tirer de ce marasme, il fallait en quelque sorte fouetter la fierté des citoyens. Ces leaders de Bouctouche ont donc privilégié le tourisme comme l'un des dossiers prioritaires de développement.

La première véritable tentative de développement touristique a été l'aménagement du Pays de la Sagouine. Cette attraction, inspirée de l' $\alpha u v r e$ littéraire d'Antonine Maillet, a été construite sur une île (1'Îleaux-Puces) accessible par une passerelle de près d'un kilomètre. La vingtaine de bâtiments qui s'y trouvent est conforme à l'architecture traditionnelle. Cette cuvre littéraire gravite autour des personnages qui a accompagnent $*$ la Sagouine et traite de l'histoire des Acadiens par des récits, des contes dans un environnement a distinctif, simple, pauve, où l'attachement à la mer est ommiprésent $\bowtie$ (LeBlanc, $1994 a: 4)$.

Les retombees du Pays de la Sagouine ont été immédiates pour la communauté puisqu'elle est passée de quelques milliers de visiteurs à une moyenne annuelle d'environ 70000 depuis 1992. Dès ses premières années d'exploitation, le Pays de la Sagouine $(48,9 \%)$ devançait le Parc national Kouchibouguac $(43,5 \%)$ et les plages $(40,9 \%)$ dans les intentions de visites des touristes de passages dans la région (LeBlanc, 1993 ; 9). Forts de ces resultats, les intervenants touristiques et économiques de la municipalité souhaitaient augmenter ces retombées. De nombreuses discussions, entrecoupées de colloques et de réunions, ont permis d'examiner diverses possibilités de développement de nature culturelle, suggérant même de faire de Bouctouche un produit d'appel culturel misant sur l'artisanat (LeBlane, 1994b : 46).

Parallèlement à ces réflexions et réagissant à la lenteur de la prise de décisions, un petit groupe de citoyens intéressés à l'environnement, mené par un chercheur

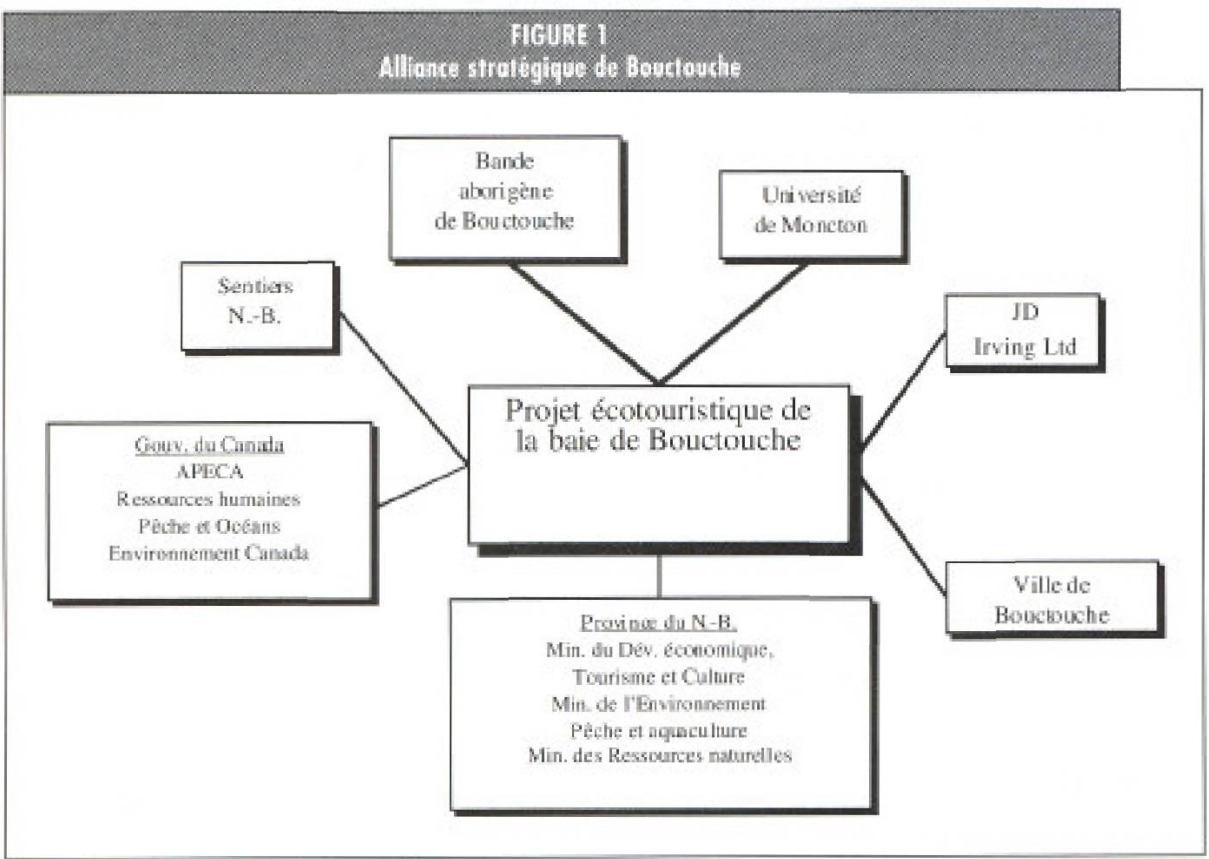

Sauree : projel écolouristique de la Baie de Bouctouche

de I'Université de Moncton $^{2}$, décidait d'assurer la protection des ressources naturelles de la municipalité. C'est au printemps 1996 que l'initiative du Projer ecotouristique de la Baie de Bouclouche a vu le jour. Le groupe avait comme objectifs de créer des emplois pour les citoyens de la ville, d'améliorer l'environnement naturel et d'encourager un épanouissement culturel (Projet écotour. de la baie de Bouctouche, 2000 : 19). De tels objectifs sont la preuve que l'intérêt reposait à la fois sur le tourisme et sur l'importance de jumeler la culture et les ressources naturelles.

\section{Le regroupement de forces vives autour d'un projet}

Un processus de près de deux ans s'est enclenché, terminé par la création et l'aménagement du complexe de l'Éco-Centre Irving de la dune de Bouctouche. Décrivons les grandes étapes de ce processus durant lequel les citoyens ont eu droit de citer dasieurs occasions. Kilcollins et Bissix (1999 : 76) précisent qu'il faut passer par cette consultation et la considérer comme l'une des étapes les plus importantes du processus de développement touristique, tout particulièrement dans le contexte cótier où la culture peut être en péril en raison d'un achalandage incontrôlé

\section{L'étape de la réflexion}

Le groupe de citoyens s'est constitué, comme le révèle la figure 1 , en a Comité directeur $*$ du projet, tout en prenant bien soin de s'adjoindre des spécialistes en environnement, en pêche, en développement durable, en tourisme, ainsi que le proprictaire de la dune, le fils de K.C. Irving ${ }^{3}$. Les membres du comité ont décidé d'embaucher une firme d'expertsconseils pour définir les grands principes du développement durable $e^{4}$ de la dune et mener une consultation publique.

Les nembres du comité ont adopté les grands principes de développement communautaire durable, c'est-à-dire l'interdépendance, la diversité, la gouvernance, l'autonomie, la rentabilite, la participation, l'égalité, l'autodétermination et une perspective $\mathrm{d}^{+}$avenir (Ashton, 2000 : 1). A 
partir de ce cadre theorique, la firme a organise trois rencontres de consultation avec les citoyens (BDA, 1996).

La première rencontre était considérée par les responsables comme une a seance d'écoute $*$ au cours de laquelle environ 200 citoyens se sont exprimés sur le développement général de leur communauté. Les discussions ont porté inévitablement sur le tourisme, en raison de l'existence du Pays de la Sagouine, et sur l'urgence de a sauver w la dune de Bouctouche. Dans le but de stimuler les discussions et de recevoir les commentaires, les responsables ont présenté les résultats de l'inventaire des ressources naturelles et touristiques. Cette rencontre a démontré que les citoyens ne voulaient pas que la communauté devienne une région reconnue pour ses plages, misant sur la baignade et attirant trop de touristes durant une courte période de temps. Ils ont exprimé le vou de maintenir une qualitế de vie acceptable dans un contexte de développement touristique axế sur l'écotourisme.

Une deuxiême rencontre (qui a attiré de 300 à 400 personnes) a eu lieu quelques mois plus tard, au cours de laquelle le plan de développement préliminaire a étế dévoilé et où l'on a encouragé les citoyens à faire part de leurs opinions. Les responsables avaient pris le soin de retenir les préoccupations ressorties lors de la première rencontre et de résumer les princi= pales idées soumises. Ils ont fait état des retombées possibles d'un développement Ecotouristique dans la ville et les environs. Les réactions obtenues lors de cette tencontre ont confirmé que le plan répondait aux aspirations de la population, ce qui a encourage les responsables à continuer leurs réflexions.

Ptès d'un an après la première rencontre, devant plus de 400 personnes, la troisième rencontre est devenue la tribune de présentation du plan final et de lancement officiel du projet écotouristique de la baie de Bouctouche.

\section{Un plan global en phase de réalisation}

L'un des principes du plan est l'identification du bassin versant et de l'estuaire de
Bouctouche comme l'ecosystème définissant cette ville. Les auteurs du plan reconnaissent \& que la santé du bassin versant de liecosysteme de la riviere de Bouctouche ainsi que la vitalite de la culure sont directement liés a la qualite de lexperience du visiteur et, par ricochet, aux possibilités offertes par l'économie touristique (Projet écotour. de la baie de Bouctouche, 2000 : 19).

L'une des premières actions menées lors de la préparation du plan a été d'analyser et de décrire les interdépendances des paysages de l'estuaire (marais salés, latgune, fleche littorale, etc.) et des habitats. Une meilleure compréhension de ce système représentait un outil essentiel au développement touristique.

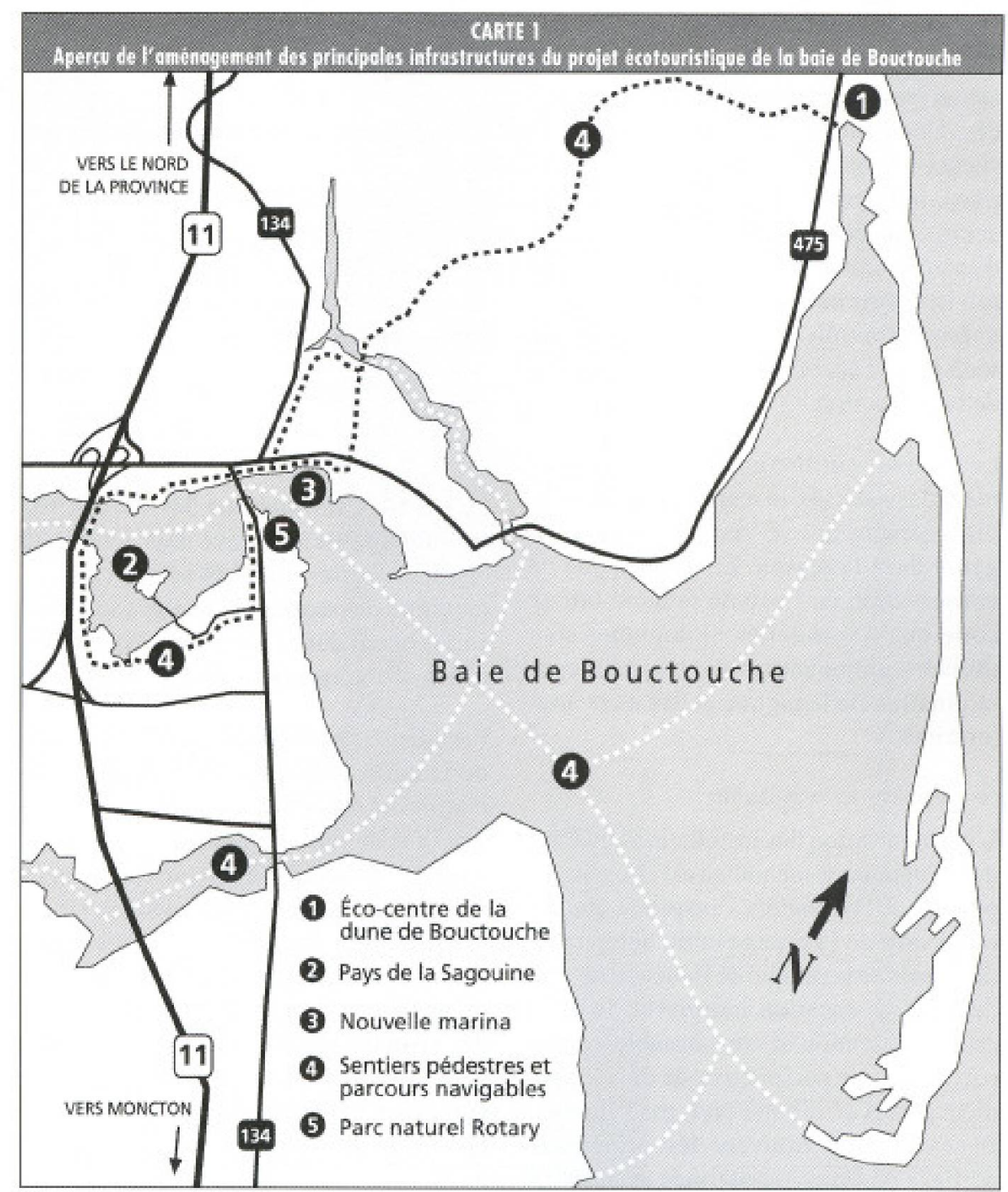

Le plan a ainsi identifié cinq projets importants d'infrastructures (décrits à la carte 1):

i) l'Éco-centre de la dune de Bouctouche,

ii) le Pays de la Sagouine,

iii) le parce le bassin de bateaux (nouvelle marina),

iv) le réseau de sentiers pédestres et de parcours navigables,

v) la restauration de l'ancien site de la marina et d'un parc naturel (Parc Rotary).

En plus de nombreux projets environnementaux dejà rếalisés (restauration du marais salé, gestion des eaux d'orages, etc.), le travail d'infrastructure des projets 
a progressé rapidement. Examinons l'état des travaux a ce jour.

\section{L'Éco-centre de la dune}

L'Éco-centre de la dune de Bouctouche a accueilli ses premiers visiteurs à l'êté $1997^{5}$. On avait alors suggéré d'utiliser des espaces précis, telle l'entrée d'un site, et de limiter les effets sur l'environnement que l'on veut protéger (Eagles, 1995), On a respectế cette consigne puisque les bâtiments de ce complexe ont été aménagés sur un ancien stationnement, minimisant ainsi les effets sur la végétation côtière fragile. On a également replanté des plantes indigènes favorisant la réintégration du site à la forêt qui l'entoure. La dune est décrite comme un superbe exemple de la beauté et des merveilles naturelless (Projet écotour. de la baie de Bouctouche, 2001 ). Un nouveau zonage de la dune, longue de $12 \mathrm{~km}$, a créé une hiérarchisation d'utilisation basée sur la fragilité de ses ressources. Une zone récréative permet aux visiteurs d'avoir accès à des activités récréatives, notamment à la plage. La zone à accès contrôlé est réservée aux activités d'interprétation de l'environnement naturel. Les chercheurs et les personnes désignées au contróle et à l'évaluation sont les seuls à avoir acoès à la troisième zone, celle de la protection.

Le complexe utilise un systeme de traitement des eaux usées respectueux de l'environnement qui se veut un modèle du genre dans la région. Le programme de conservation de l'eau de la dune fait en sorte que des toilettes biologiques sont utilisées, éliminant ainsi le risque de contamination de la lagune et des eaux souterraines.

\section{Le Pays de la Sagouine}

L'harmonisation des installations du Pays de la Sagouine à son milieu est une réussite puisque ses bâtiments s' inspirent de l'architecture de la grange et du hangar traditionnels de la ruralité de Bouctouche. Audelà de sa vocation culturelle, le Pays retient l'attention des responsables, d'une part, dans une perspective de développement global et, d'autre part, par l' ̂̂le-auxPuces - où se trouvent les principaux édifices du Pays - constituée de spartine alterniflore indigène, d'épinettes indigènes, d'églantines et de puccinel.

Le parc et le bassin de bateaux

(nouvelle marina)

On a aménagé une nouvelle marina qui s'intègre au plan de développement dans 1a mesure où elle vise essentiellement à fournir un bassin pour les opérateurs de tours guidés et pour la location de canots et de bateaux. Ce bassin servira à rétablir le « marais salant à partir d'une replantation d'especes natives du milieu * (Projet écotour, de la baie de Bouctouche, 2001).

Le réseau de sentiers pédestres et de parcours novigables

Le travail de restauration du paysage riverain se poursuit avec l'établissement d'un réseau de sentiers de l'arriè̀ baie qui relie le Pays de la Sagouine, le centre-ville et ếventuellement l'Éco-centre de la dune. Le sentier s'étend sur une longueur de $17 \mathrm{~km}$. Durant les travaux d'aménagement, on a reboisé certains endroits avec des arbres indigènes. Cette restauration servira aussi de filtre des eaux aux abords de la rivière, de corridor pour la faune et de sentier récréatif pour l'agrément des rétsidents et des touristes.

La restauration de l'ancien site de la marina et d'un parc naturel (Parc Rotaryl

Le Centre d'information des visiteurs de la municipalité se trouve déjà sur ce site en plein centre-ville. Les travaux de restauration des marais salants se sont poursuivis et ont donné lieu à la création d'un centre d'interprétation sur le sujet.

Par ailleurs, mentionnons que l'Éco-centre de la dune, le Pays de la Sagouine et la marina ont été construits sur pilotis, ce qui a permis de réduire les effets sur le drainage de surface et d' $c$ viter des dommages permanents qui pourraient decouler de la construction. En outre, de nombreuses terrasses et des plates-formes de bois permettent de contrôler les allées et venues des visiteurs et d'éviter qu' ils endommagent la végétation. Comme le suggèrent Covey et Flanagan, tous les bâtiments épousent une architecture qui s 'intègre bien au paysage naturel (1992: 153),

\section{Des retombées sur la fierté}

L'observateur averti n'aura aucune difficulté à témoigner des changements résultant du développement de la dune et du bassin versant de la baie de Bouctouche au cours des dernières années. Ces retombées peuvent être regroupées en trois catégories : environnementales, communautaires et économiques.

\section{Les retombées environnementales}

Rien de surprenant que les retombées environnementales viennent en tête de liste puisqu' il s'agit de la raison d'être de toute l'initiative de la communauté. Les différents projets et la construction d' infrastructure prennent leur origine dans la préservation et la restauration des richesses naturelles de la baie et du bassin versant dans leur ensemble. Ce sont d'ailleurs des questions liếes à l'environnement qui ont motivé des leaders à se regrouper et à réaliser le projet. Très vite, la communauté s'est sentie interpellée et a répondu favorablement à la vision que voulait lui donner ce groupe :

- créer une économie touristique axée sur le marché écotouristique et fondée sur la culture et l'environnement unique de Bouctouche ;

- intégrer la nouvelle économie touristique à d'autres secteurs de l'économie, en particulier, I'industrie de la culture des huîtres:

- s'assurer de maintenir l'intégritế écologique $^{t}$ de la région de Bouctouche ;

- maximiser les possibilités économiques pour les collectivités locales (Projet ecotour. de la baie de Bouctouche, 2000 : 48).

Même si la protection de l'environnement gagne en notoriété dans les régions des pays industrialisés et developpés, la population de Bouctouche semble vouloir épouser ce concept ; elle a la chance de l'observer et de le mettre en pratique. Un sondage réalisé en 2000 par une firme indépendante auprès de 449 résidants de la ville a permis de confirmer l'intérêt des citoyens pour la cause environnementale 
(Planning@Work, 2000). Voici un aperçu des principaux résultats obtenus:

- $80 \%$ des personnes interrogées se prononcent favorablement sur les développements de la région au cours des trois à cinq dernières années. Parmi ces développements, on note les sentiers, l'Éco-centre de la dune, les nouvelles entreprises et, d'une manière générale, la participation des citoyens;

- $70 \%$ des répondants ont exprimé leur inquiétude face à l'environnement et aux aires naturelles;

- 90 \% considèrent qu'une gestion de croissance plus efficace du bassin versant constitue une priorite :

- $75 \%$ souhaitent une utilisation efficace des ressources du territoire en premier lieu. La protection des sites et des edifices historiques obtient le même appui (75\%).

\section{Les retombées communautaires}

L'ensemble des retombées communautaires $n^{\prime}$ a pas encore été evalué par les responsables du projet. Toutefois, comme le démontre les résultats du sondage (Planning@Work, 2000), la population semble exprimer un sentiment de fierté face à ce qui s'est passé dans leur ville. Ajoutons à ceci la participation importante aux rencontres publiques $(200$ a 400 personnes sur une population totale de 2500 ) au début du processus de planification.

L'une des principales retombées communautaires a été la création d'un programme de formation destiné aux autres communautés s ${ }^{\dagger}$ intitulant * Planifier une destination touristique communautaire durable : Bâtir à partir de l'expérience de Bouctouche $*$. Cette initiative a reçu l'aval et du financement de l'Agence de promotion economique du Canada Atlantique.

Le programme utilise la formule du séminaire de deux jours basée sur une expérience interactive. Il est destiné aux dirigeants communautaires qui veulent en apprendre davantage sur les pratiques de développement durable d'une destination touristique. Parmi les sujets abordés durant la formation, on note l'encouragement du sens de leadership chez les dirigeants communautaires, la participation communautaire, les ressources financières, la gestion du processus de recherche, l'évaluation du potentiel touristique, les principes du développement durable, les alliances, les partenariats et la gestion des ressources.

Le programme est offert par des spécialistes en environnement, en tourisme et en développement communautaire qui se sont intéressés de près au projet de Bouctouche. Lancé à l'automne 2000 , le programme a déjà attiré une demi-douzaine de municipalités ei de régions pour des sessions, en langue française ou en langue anglaise. Pour l'instant, les communautés intéressées proviennent surtout du littoral acadien et de la baie de Fundy.

Tout en reconnaissant que leur projet n'est pas, malgré son succès, la formule magique et parfaite pour d'autres villes et villages, les responsables manifestent une certaine fierté à partager leurs expériences avec d'autres leaders. Ils ont pris grand soin de ne pas faire du programme une description pure et simple de leur projet. Plutôt, à partir des expériences tirées, ils ont conçu un programme complet dont les assises théoriques sont solides et qui comprend des exercices pratiques sur le terrain en plus d'exposer les visiteurs à l' hospitalité acadienne et aux produits culinaires régionaux.

Les retombées communautaires s'expriment également dans la sensibilisation de la population au concept de développement durable. Les auteurs du sondage déclarent, quand ils résument l'ensemble du succès du projet écotouristique, que :

Le développement durable requiert un progrès au niveau de l'économie et de l'emploi, ainsi qu'un respect de l'environnement et une conscience sociale. Les résidents s'inquiètent au sujet de l'économie et de l'emploi. C'est un élément essentiel au développement. IIs se soucient également de l'environnement et désinent s'impliquer dans la planification future de la communauté. Les efforts déployes pour conscientiser les gens du bassin versant aux activites, aux développements ainsi qu'à leur im- plication doivent être maintenus (Planning@Work, 2000 : 3).

\section{Les retombées économiques}

Les responsables du projet, dans le souci de montrer aux citoyens toutes les facettes des retombees de leurs actions, ont donc colligé une série de donnés économiques depuis 1996 (présentées au tableau 1).

L'achalandage du Centre d'information des visiteurs a littéralement éclaté avec une augmentation de $185 \%$. Le nombre de visiteurs du Pays de la Sagouine est passé de 63000 à $87000(+36 \%)$ et l'Éco-centre de la dune a connu une augmentation de visiteurs de $85 \%$, de 1997 à 1999.

D'une municipalité avec un niveau quasi inexistant d'investissements en infrastructure touristique dans les années 1980 , Bouctouche a bénéficié d'investissements, essentiellement gouvernementaux, de l'ordre de 12 à 15 millions de dollars de 1996 à 1999. La famille Irving a investi une partie importante de ces millions ${ }^{7}$. A cela, il faut ajouter une somme d'environ 4 millions de dollars pour la création du Pays de la Sagouine au début des années 1990. Le secteur privé a manifesté sa confiance en investissant plus de 4,4 millions de dollars. Le nombre d'entreprises touristiques a augmenté de 45 de 1996 à 1999. Un total de 116 nouveaux emplois ont été crés, totalisant l'équivalent de 76 personnes-années durant la même période.

Les données préliminaires de l'année 2000 révèlent une diminution de l'achalandage de $38 \%$ au Centre d'information des visiteurs ( 8413 visiteurs en 2000 ) et de $24 \%$ pour l'Éco-centre de la dune (117 797 visiteurs en 2000). Le Pays de la Sagouine a connu une diminution de $9 \%$ avec 79253 visiteurs. Ces baisses s'expliquent en grande partie par la diminution généralisée à l'ensemble de la province du Nouveau-Brunswick (Comm. 6conom. de Kent, 2000). Aucune compilation n'a encore été réalisée pour le nombre de nouvelles entreprises, la création d'emplois et les investissements.

La municipalité et l'ensemble de la région ont benéficié de retombées importantes du 


\begin{tabular}{|c|c|c|c|c|c|}
\hline \multicolumn{6}{|c|}{ 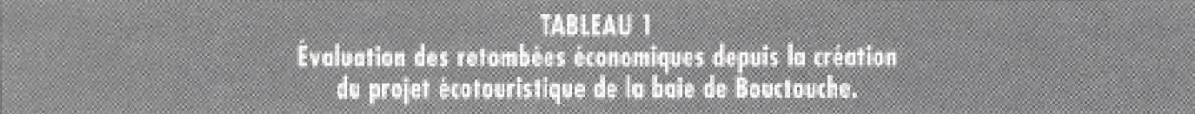 } \\
\hline $\begin{array}{l}\text { Investissement direct dans } \\
\text { l'infrastructure des projels }\end{array}$ & \multicolumn{4}{|c|}{1996.1999} & Détails \\
\hline 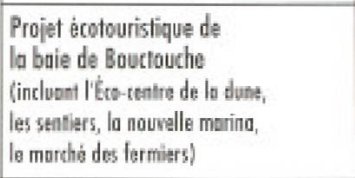 & \multicolumn{4}{|c|}{8 millions $\$$} & $\begin{array}{l}65 \% \text { - Sources locoles } \\
20 \% \text { - Gour prow } \\
15 \% \text { - Gour fed. }\end{array}$ \\
\hline $\begin{array}{l}\text { Poys da la Sagouine } \\
\text { (phase drexponsion - 10909] }\end{array}$ & \multicolumn{4}{|c|}{1 million $\$$} & $\begin{array}{l}85 \% \text { - Goun féd. } \\
15 \% \text { - Gouv prow. }\end{array}$ \\
\hline 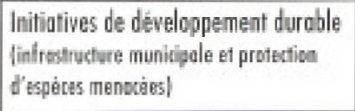 & \multicolumn{4}{|c|}{3 a 5 millions $\$$} & Plusieurs portenoires \\
\hline Fréquentation des visiteurs & 1996 & 1997 & 1998 & 1999 & Variations \\
\hline Centre d'information des vișteurs & 4750 & 9253 & 11957 & 13533 & $7185 \%$ \\
\hline Poys de la Sogauine & 63903 & 72943 & 87817 & 87086 & $736 \%$ \\
\hline $\begin{array}{l}\text { Exo-centre de la dune } \\
\text { (ouverture en 1997) }\end{array}$ & - & 88500 & 187691 & 163947 & $\boldsymbol{\pi}_{85}$ \\
\hline $\begin{array}{l}\text { Créations d'entreprises ef } \\
\mathrm{d}^{\prime} \text { emplois du secteur privé }\end{array}$ & 1996 & 1997 & 1998 & 1999 & Total \\
\hline Mouvelles entreprises touristiques & 4 & 9 & 14 & 18 & 45 \\
\hline Investissements prives & $440000 \$$ & 18900005 & 6340005 & $1476000 \$$ & $4440000 \$$ \\
\hline Emplois du secteur touristique & 321 & 389 & 415 & 437 & $\begin{array}{l}\text { T116 } \\
\text { newebox atiplosis de } \\
1996 \text { a } 1997\end{array}$ \\
\hline Emplois en personnes-années & 128 & 170 & 190 & 200 & $\begin{array}{l}772 \\
\text { enplois pesonnes-uninies } \\
\text { de } 1996 \text { a } 1997\end{array}$ \\
\hline
\end{tabular}

Source : Projer ecotouristiqua de la baie de Bouctouche Ine.

projet écotouristique qui misait sur les richesses de son littoral. Que faut-il faire maintenant pour continuer sur cette lancé ?

\section{Devenir un modèle est difficile, mais le demeurer est un défi tout aussi important}

Conscients de leurs réussites, les responsables reconnaissent que le défi le plus difficile est à venir, c' est-à-dire s'assurer de continuer sur la lancée des années 1990. L'intérêt de la population est toujours palpable, mais les ressources sont de plus en plus difficiles à obtenir et on dénote qu'un certain essoufflement des leaders commence à se faire sentir. Heureusement, les responsables sont lucides et veulent éviter que l'enthousiasme ne cède au découragement devant la tâche à accomplir. Ils ont donc entrepris une série de mesures concrètes dont voici les principales.

\section{Établir une structure \\ de gestion permanente}

Des tentatives de regroupement des entreprises et des intervenants sont présente- ment en cours dans le but principal de coordonner les efforts de marketing. Au-delà des efforts pour attirer les écotouristes, les leaders veulent s'assurer que les touristes culturels (attirés par la culture acadienne et le Pays de la Sagouine) savent ce qui est offert dans la région immédiate de Bouctouche. Il s'agit d'ailleurs d'une des prémisses pour attirer ce type de touriste, selon McKay, qui explique que le touriste est plus intéressé à se déplacer pour les «attributs \& de la région que pour l'acte de voyager (1999: 155).

Il est clair pour les dirigeants qu'il est essentiel qu'ils s'organisent sur le plan local avant d'intégrer complètement les autres paliers d'organisation touristique (régionaux et provinciaux). Comme le souligne Johnson dans son analyse des organisations localles du tourisme au Québec : * Les organisations locales de tourisme répondent aux besoins des collectivités locales de prendre en main leur développement. En intervenant sur des territoires restreints, les organisations locales per- mentent de concilier plus facilement les intérêts de différents intervenants qui partagent le même cadre de références. (2000:19).

Pour l'instant, le chaînon manquant est le gouvernement local. Le conseil municipal a appuyé le projet, mais semble très réticent à y investir davantage de ressources. Une municipalité de 2500 habitants est effectivement limitée dans ses actions visant à aider un projet de développement de ce type. Il n'empêche qu'elle peut assurer un leadership déterminant pour aller justement chercher ailleurs l'aide souhaitée. En examinant la situation, il ne semble pas évident que la municipalité a assuré un leadership à la hauteur du projet. Reconnaissons pour autant qu'elle ne l'a pas non plus entravé...

\section{Assurer une évaluation continue des progrès}

Peu importe la forme de la structure qui sera mise en place, les responsables insistent sur la vigilance continuelle des retombées du projet. Ils souhaitent mesurer les progrès environnementaux en matière de restauration et de protection, tout comme les retombé é conomiques et communautaires. Ils maintiennent comme l'une de leurs priorités l'importance d'informer la population de l'état des travaux et de leurs retombées. La transparence du projet et les renseignements fournis à la population ont jusqu'à maintenant assuré une bonne partie du succès. Ces bonnes relations doivent se poursuivre, puisque le développement de la municipalité en ressortira gagnant.

Les leaders ont pris les choses en main jusqu'à maintenant, en s'assurant de laisser une grande place à la population locale et ils ont vu les fruits de leurs labeurs récompensés concrètement par des retombées environnementales, communautaires et économiques considérables. C'est grâce en grande partie à leur travail acharné que la ville de Bouctouche a été reconnue en 2000 comme l'une des dix plus belles villes canadiennes par un magazine spécialisé (Gilford, 2000). Un clin d' $\alpha$ il anecdotique, mais qui fait sûrement plaisir !

À partir de l'expérience positive du cas de Bouctouche, peut-on considérer que le 
tourisme est au service de la communauté ou plutôt que la communauté est au service du tourisme? Reconnaissant que cette hypothèse de départ est ambitieuse et difficile à circonscrire sans d'autres démarches de recherches, il semble que la communauté de Bouctouche a le contrôle de sa destinée par l'écoute et le respect des leaders engagés dans ce projet. Jusqu'à présent et malgré les défis à relever dans l'avenir, les démarches laissent une place importante à la participation populaire et à la prise de décision, tout en tenant compte des préoccupations des citoyens.

\section{Mare LeBlane est professeur agrégé} a l'Ecole de kinésiologie et de récréologie del'Universite de Moncton au NouveauBrunswick.

\section{Notes}

1 Le développement durable $*$ abreuve $*$ la philosophie de l'écotourisme selon Nadeau. Il précise, entre autres, qu'on peut considérer les concepts de tourisme vert, de tourisme écologique, d'ecotourisme et de a sustainable development s comme une * rendance lourde a l'échelle mondiale *, proposant * whe approche nowvelle da phénomène touristique, résolument plus respectueuse du milieu d'accueil s (1999: 73).

2 Il s'agit de Louis Lapierre, environnementaliste de réputation enviable dans la région et en Amérique du Nord. Les responsables du projet le considèrent comme un visionnaife et le a champion * nécessaire à la réussite d'une telle entreprise.

3 Le rôle du fils de K.C. Irving, J.K. Irving. a été déterminant tout au long du processus et il l'est toujours aujourd'hui. Cet homme d'affaires, en plus d'engager d'importantes sommes d'argent, a agi comme élément déclencheur de nombreuses décisions communautaires et politiques. Il s'est constamment assuré d'avoir l'appui des citoyens de la Ville de Bouctouche. Il disait et répète encore que les citoyens doivent prouver qu'ils veulent se prendre en main et que de son côté il fera sa part. Ce milliardaire fait de ce projet son héritage pour la communaté de Bouctouche qui a vu naître et grandir son père.

4 Les responsables et les auteurs de l'étude ont retenu la définition d'Environnement Canada (1995), c'est-à-dire : « Le développentent durable foumit un cadre theorigue pour l'integration de politiques environne- mentales et de stratégies de développéments. Le développement durable recon. natt que la progression est essentielle pour satisfaire les besoins et la qualité de vie de I'humain. Cependant, le developpement doit se faire de façon la plus efficace, écologique et responsable possible des ressources naturelles, humaines et économiques $\%$.

5 La photo ci-contre date de 1997. Depuis lors, la configuration de la dune a été modifiée en raison des intempéries, notamment lors d'une tempête à l'automne 2000 . Heureusement, il n'y aura pas d'effets importants sur l'évolution de l'habitat.

6 La lecture des différents travaux sur ce projet démontre que le concept d'intégrité écologique est inclusif à une vaste démarche qui comprend l'ensemble des êlements. naturels de la région.

7 La famille Irving est toujours très discrète quant à l'ampleur de ses affaires. On peut néanmoins estimer à 4 ou 5 millions de dollars son investissement dans l'aménagement des infrastructures du Projet écotouristique de la baie de Bouctouche.

\section{Bibliographie}

Ashton, Bill (2000), Communautes rurales durables... On y woit ?, Bouctouche, NouveauBrunswick.

BDA, (1996), Plan d'écotourisme de la Baie de Bouctouche, Sussex, Nouveau-Brunswick.

Commission économique de Kent Ine. (2000), Centres d'information aux visiteurs : Region de Kent, Bouctouche, Nouveau-Brunswick.

Covey, Frank J, et Sheila Flanagan (1992), \& Tourism and the Environment-Impact and Solutions *, in Helen Briassoulis, et Jan van der Straaten (Ed.), Tourism and the Environment: Regional Economic and Policy Issues, p. 145153.

Eagles, Paul F. (1995), o Understanding the Market for Sustainable Tourism *, in Stephen McCool, et Alan Watson (Ed.), Linking Tourism, the Environment and Sustainability, http// www.ecotourism.org/datafr.html.

Environnement Canada (1995), Guide pour un gouvernement vert, Ottawa, Gouvernement du Canada.

Gilford, Eric (2000), * My Kind of Town *, Harrowsmith Country Life, avril, p. 82-83.

Gouvernement du Nouveau-Brunswick (2000), Bienvenue au Nonveau-Brunswick 2000 , Fredericton, Nouveau-Brunswick.

Gunn, Clare, A. (1972), Vacationscape: Designing tourism regions, New York, Van Norstrand Reinhold.

Johnson, Lynda (2000), * Les organisations locales et régionales en tourisme s, Téoros, 19 (3), $\mathrm{p}, 15-19$.
Kilcollins, Adam, et Glyn Bissix (1999), * The Potential Impact of Ecotourism and Nature Tourism Development on a Coastal Community: The Case of Cheticamp, Inverness County, Cape Breton, NS s, in Paul Heintzman (Ed.), Leisure, Politics and Power: Driving the Social Agenda into 21 st Century, p. 75-77.

Lack, Kathryn (1999), \& La Dune de Bouctouche: Habitat Protection through Research and Visitor Information $w_{\text {, in }}$ Canadian Tourism Commission and Center for Tourism Policy Research, Simon Fraser University, On Route to Sustainability, Best Practice in Canadian Tourism, p. 110-116.

LeBlanc, Marc (1993), Résultats de l'enquête menée auprès des visiteurs des Centre d'intormation touristique de la région de Kent durant I'été 1993, Dieppe, Nouveau-Brunswick.

LeBlane, Marc (1994a), Relancement du a produit " Pays de la Sagouine : Plan de marketing, Moneton, Nouveau-Brunswick.

LeBlanc, Marc (1994b), Evaluation des infrastructures touristiques de la région de Kent, Dieppe, Nouveau-Brunswick.

McHarg, Ian (1969), Design With Nature, New York, Garden City Press.

McKay, Kelly (1999), a Heritage Tourists: Profiling an Important Segment of Provincial Travelers s, in Paul Heintzman (Ed.), Leisure, Politics and Power: Driving the Social Agenda into 2 Ist Century, p. 153-156.

Nadeau, Roger (1999), \& Tourisme et environnement $x$, in Normand Cazelais, Roger Nadeau, et Gerard Beaudet (Ed.) L Lespace touristique, p. 61-131,

Planning@Work (2000), La Commuraste se prononce ?, Bouctouche, Nouveau-Brunswick.

Potts, Thomas D., et Rich Harrill (1998), * Enhancing Communities for Sustainability: A Travel Ecology Approach *, Tourism Analysis, 3 (3-4), p. 133-142.

Projet écotouristique de la baie de Bouctouche Inc. (1999), Sondage du profil des visiteurs de Bouctouche, Bouctouche, Nouveau-Brunswick.

Projet écotouristique de la baie de Bouctouche Inc. (2000), Planifier une destimation touris. tique communataire durable : Batir à partir de lexperience de Bouctouche, $N_{-}-B$. Bouetouche, Nouveau-Brunswick.

Projet écotouristique de la baie de Bouctouche Ine, (2001), http:/www.ecotourismnb.com.

Savoic, Donald (1992), Regional Economic Development: Canada's Search for Solutions, Toronto, University of Toronto.

Seekings, Jim (1980), \& Pro Bono Publico: The Case for a Systematic System *, in D.W. Hawking, E.I. Shaffer, and J.M. Rovelstad (Ed.), Tourism Planning and Development lssues, p. 251-257. 